\title{
Características de imagem na ressonância magnética de gossipiboma intracraniano: relato de caso e revisão da literatura*
}

\author{
Magnetic resonance imaging findings of intracranial gossypiboma: a case report \\ and literature review
}

Michele Carneiro Brito Martins ${ }^{1}$, Raquel Portugal Guimarães Amaral ${ }^{2}$, Celi Santos Andrade ${ }^{3}$, Leandro Tavares Lucato ${ }^{4}$, Claudia da Costa Leite ${ }^{5}$

\begin{abstract}
Resumo Vários trabalhos têm descrito as características de gossipibomas por meio de radiografias convencionais e tomografia computadorizada, porém, seus achados na ressonância magnética são menos conhecidos. 0 objetivo deste estudo foi descrever as características de imagem de gossipibomas, ilustrando um caso de uma paciente com quadro clínico de febre e confusão mental com antecedente de cirurgia encefálica.

Unitermos: Imagem por ressonância magnética; Encéfalo; Granuloma; Gossipiboma.
\end{abstract}

Abstract Many reports in the literature have described findings of gossypibomas at conventional radiography and computed tomography. However, magnetic resonance imaging findings are still to be completely known. The purpose of the present study was to describe imaging findings of gossypibomas in a patient with previous history of brain surgery, presenting fever and mental confusion.

Keywords: Magnetic resonance imaging; Brain; Granuloma; Gossypiboma.

Martins MCB, Amaral RPG, Andrade CS, Lucato LT, Leite CC. Características de imagem na ressonância magnética de gossipiboma intracraniano: relato de caso e revisão da literatura. Radiol Bras. 2009;42(6):407-409.

\section{INTRODUÇÃO}

Vários termos são utilizados na literatura médica para descrever um processo inflamatório relacionado a corpo estranho ${ }^{(\mathbf{1})}$. O termo "gossipiboma" é usado para descrever uma massa formada a partir de

* Trabalho realizado no Setor de Ressonância Magnética do Instituto de Radiologia do Hospital das Clínicas da Faculdade de Medicina da Universidade de São Paulo (InRad/HC-FMUSP), São Paulo, SP, Brasil.

1. Médica Radiologista, Especializanda do Setor de Ressonância Magnética do Instituto de Radiologia do Hospital das Clínicas da Faculdade de Medicina da Universidade de São Paulo (InRad/HC-FMUSP), São Paulo, SP, Brasil.

2. Médica Radiologista, Pesquisadora do Setor de Ressonância Magnética do Instituto de Radiologia do Hospital das Clínicas da Faculdade de Medicina da Universidade de São Paulo (InRad/HC-FMUSP), São Paulo, SP, Brasil.

3. Médica Radiologista, Prática Profissionalizante do Setor de Ressonância Magnética do Instituto de Radiologia do Hospital das Clínicas da Faculdade de Medicina da Universidade de São Paulo (InRad/HC-FMUSP), São Paulo, SP, Brasil.

4. Doutor, Vice-chefe do Setor de Ressonância Magnética do Instituto de Radiologia do Hospital das Clínicas da Faculdade de Medicina da Universidade de São Paulo (InRad/HC-FMUSP), São Paulo, SP, Brasil.

5. Professora Associada do Departamento de Radiologia Chefe do Setor de Ressonância Magnética do Instituto de Radiologia do Hospital das Clínicas da Faculdade de Medicina da Universidade de São Paulo (InRad/HC-FMUSP), São Paulo, SP, Brasil.

Endereço para correspondência: Dra. Michele Carneiro Brito Martins. Rua Bela Cintra, 2206, ap. 73, Cerqueira César. São Paulo, SP, Brasil, 01415-002. E-mail: mcbbrito@hotmail.com

Recebido para publicação em 23/1/2008. Aceito, após revisão, em 5/6/2008. uma matriz de algodão cercada por uma reação inflamatória/granulomatosa ${ }^{(\mathbf{1})}$. A palavra é derivada do latim gossypium (algodão) e do kiswahili* boma (lugar de dissimular, ocultar) ${ }^{(2)}$. Outros termos utilizados são textiloma, derivado do latim textile (tecido) e associado ao sufixo oma (que significa tumor ou edema), gauzoma, de gazes cirúrgicas, e muslinoma, referente a tecido de musseline ${ }^{(\mathbf{1})}$.

Embora alguns materiais possam ser visualizados prontamente usando a radiologia convencional e a tomografia computadorizada (TC), graças aos filamentos radiodensos no seu interior, os padrões na ressonância magnética (RM) são menos conhecidos. Eventualmente, pequenos fragmentos de material cirúrgico podem não conter filamentos radiodensos, dificultando sua identificação direta pelos métodos de imagem por emissão de raios $\mathrm{X}$, sendo a RM uma ferramenta de extrema utilidade nestes casos ${ }^{(\mathbf{3 , 4})}$. Além disso, existem materiais bioabsorvíveis que também podem desenvolver reação inflamatória e que não são radiodensos.

* Dialeto do interior da Tanzânia.
Uma reação inflamatória exuberante, dependendo da história clínica de cada paciente, pode ser confundida com inúmeras doenças, como tumores recorrentes, radionecrose, abscessos, hematomas, infartos em resolução, tumores primários ou metástases $^{(\mathbf{1})}$. Para que este erro não ocorra, salientamos a importância de estarmos familiarizados com a aparência de um gossipiboma intracraniano nas imagens de RM.

No presente relato descrevemos o padrão de imagem na RM de um gossipiboma intracraniano, o qual, comparado com gossipibomas encontrados após cirurgias abdominais e ortopédicas, é relativamente raro $^{(5)}$. O objetivo deste relato é alertar para esta possibilidade diagnóstica e fazer uma revisão da literatura sobre a terminologia e os materiais utilizados no intraoperatório.

\section{RELATO DO CASO}

Paciente do sexo feminino, 33 anos de idade, com diagnóstico prévio, há seis anos, de neurocisticercose intraparenquimatosa supratentorial e racemosa na fossa posterior, comprometendo o quarto ventrículo. Foi submetida a quatro cirurgias nos 
últimos dois anos, a última há 20 dias, na região da fossa posterior. Evoluiu, no pós-operatório tardio, com febre, diminuição do nível de consciência e secreção purulenta pela cicatriz cirúrgica.

Foi solicitada TC, que evidenciou formação expansiva na fossa posterior com realce intenso após contraste. Realizou-se RM, que demonstrou conteúdo com hipossinal em T1 na fossa posterior, obliterando o quarto ventrículo, com focos de maior hipossinal compatíveis com ar/gás. Estes focos também estavam presentes no trajeto cirúrgico, na região póstero-lateral direita (Figura 1). Após a administração de contraste houve realce nas margens da lesão e meninges adjacentes à craniotomia (Figura 2). Na imagem sagital T1 após contraste foi possível identificar a continuidade da lesão com o trajeto cirúrgico, estendendo-se para a região póstero-lateral direita da fossa posterior, assim como os focos de marcado hipossinal (ar/gás) (Figura 3). Na sequência coronal T2 a lesão apresentava hipersinal heterogêneo com focos de marcado hipossinal no interior e áreas liquefeitas periféricas (Figura 4). No parênquima cerebelar adjacente observava-se hipersinal em T2 e FLAIR, assim como na ponte, mesencéfalo, pedúnculos cerebelares. A sequência de difusão evidenciou restrição à difusão das moléculas de água em alguns pontos da lesão.

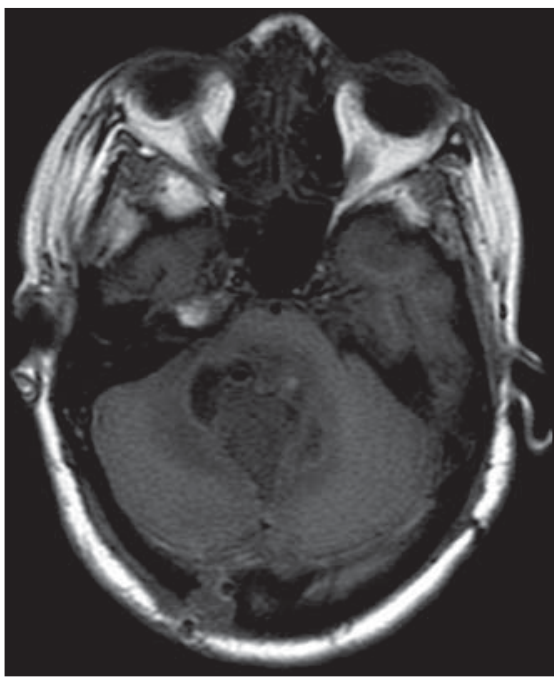

Figura 1. Imagem axial T1 mostra conteúdo com hipossinal localizado na fossa posterior obliterando o quarto ventrículo, com focos de marcado hipossinal que podem corresponder a ar/gás, também identificados próximos à região da craniotomia.

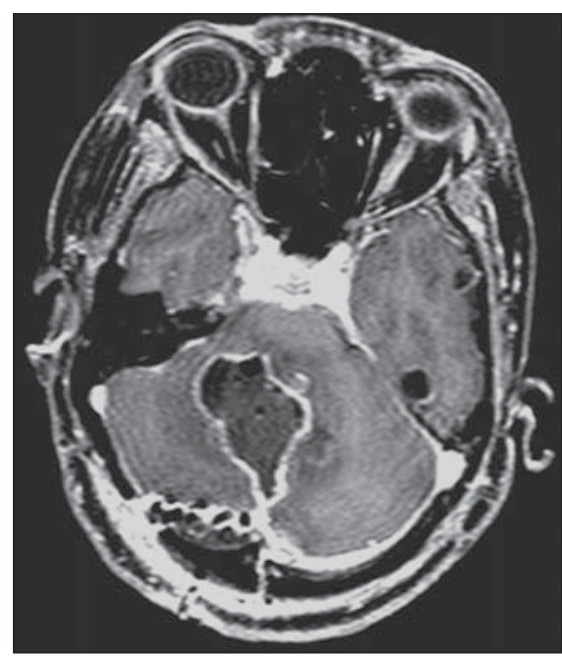

Figura 2. Imagem axial T1 com contraste evidencia intenso realce nas margens do sítio cirúrgico e lesão hipodensa obliterando o quarto ventrículo, sem realce. Existem focos de marcado hipossinal no interior da lesão e no trajeto cirúrgico, mais evidentes na região posterior direita, já identificados no exame sem contraste, que podem corresponder a ar/gás.

Estas características, associadas à história clínica, sugeriram fortemente a possibilidade de processo inflamatório/granulomatoso com conteúdo no interior do sítio cirúrgico, podendo corresponder a corpo estranho (gossipiboma).

Após a cirurgia, a hipótese diagnóstica foi confirmada, encontrando-se fragmento de gaze envolvido por processo inflamatório/granulomatoso. Portanto, seria mais correto usar o termo gauzoma do que gossipiboma, já que este último é mais genérico.

\section{DISCUSSÃO}

Uma grande variedade de agentes hemostáticos é usada para evitar hemorragias durante e após o ato cirúrgico. São utilizados materiais não reabsorvíveis e absorvíveis. Exemplos de materiais não absorvíveis são gazes de algodão (gauzoma), materiais sintéticos de rayon ou retalhos de musselina (muslinoma). Já os materiais absorvíveis mais utilizados são: espuma de gelatina $\left(\right.$ Gelfoam $\left.^{\circledR}\right)$, celulose oxidada (Surgicel $^{\circledR}$, Oxycel $^{\circledR}$ ) e colágeno microfibrilar (Avitene $\left.{ }^{\circledR}\right)^{(\mathbf{1})}$, que são deixados no local de manipulação para se evitar hemorragias de repetição.

Existem também materiais sintéticos utilizados para substituir defeitos na dura-

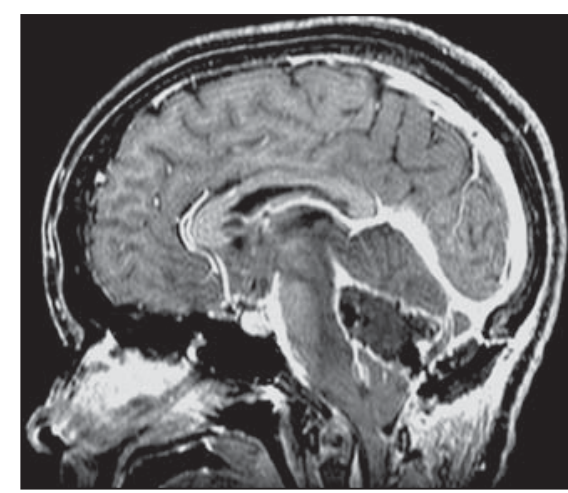

Figura 3. Imagem sagital T1 com contraste, em que fica mais bem demonstrada a continuidade dos focos de marcado hipossinal adjacente à craniotomia, com o sítio cirúrgico (ar/gás) e no interior deste conteúdo/lesão heterogênea.

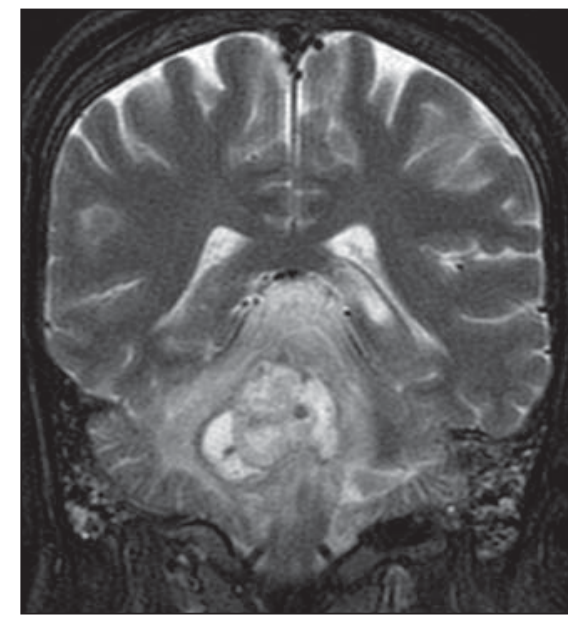

Figura 4. Imagem coronal T2 evidencia hipersinal heterogêneo do conteúdo na fossa posterior, com focos de marcado hipossinal (ar/gás) e áreas liquefeitas de hipersinal perifericamente. Há também sinais de mastoidopatia bilateral.

-máter $\left(\right.$ Silastic $\left.^{\circledR}\right)$, materiais quimioterápicos (bolachas de Gliadel ${ }^{\circledR}$ ), outros utilizados como agentes de embolização para tumores altamente vascularizados (Gelfoan ${ }^{\circledR}$ e Avitene $\left.{ }^{\circledR}\right)$, e microesferas de tris-acril gelatina e partículas de álcool polivinil (emboesferas) $^{(\mathbf{1})}$.

Eventualmente, materiais não reabsorvíveis são "esquecidos" no sítio cirúrgico. Todos eles podem desencadear uma reação inflamatória ${ }^{(1)}$. O material não reabsorvível induz a dois tipos de reação ${ }^{(6)}$. Uma reação é exsudativa quando leva à formação de abscesso com ou sem infecção bacteriana secundária. $\mathrm{O}$ processo inflamatório pode ocorrer no primeiro dia e a reação granulomatosa, após uma semana. Se o material 
se infectar, um abscesso é formado e, consequentemente, uma fístula cutânea para drenagem ${ }^{(3,7)}$. Já os agentes absorvíveis desencadeiam um processo inflamatório fisiológico até que ocorra a absorção completa do material. Na maioria dos casos este processo é assintomático.

Antigamente, a reação inflamatória envolvendo um material hemostático era chamada de textiloma. Entretanto, ele deve ser usado apenas para materiais orgânicos ${ }^{(5,7)}$. Materiais sintéticos associados ao algodão estão sendo amplamente utilizados, portanto, o termo textiloma foi substituído por "gossipiboma"(6,8). Além disso, o termo textiloma está amplamente associado a cirurgias gerais, cujo material, deixado iatrogenicamente, motivou processos médico-legais. No entanto, existem autores que defendem o uso do termo textiloma, por sua consagração pelo uso ${ }^{(\mathbf{1})}$.

Formas assintomáticas são descobertas acidentalmente meses ou anos após a cirurgia $^{(\mathbf{9})}$. No presente relato, a paciente apresentou sintomas 20 dias após a cirurgia. $\mathrm{O}$ tipo exsudativo com processo inflamatório bacteriano causa sintomas mais precocemente que o tipo fibrótico ${ }^{(\mathbf{1 0})}$.

Os gossipibomas apresentam aparência radiológica inconstante, que depende do tempo em que o corpo estranho está no sítio cirúrgico, do tipo de material utilizado e da localização anatômica. O sinal da RM depende da quantidade de fluido e proteína associada à lesão.

Estudos anteriores mostraram que a imagem na RM é de massa bem circuns- crita, com hipossinal em T1 e hipersinal em $\mathrm{T} 2^{(3,7)}$. Possui uma cápsula com marcado hipossinal em T1 e T2. Porém, outras publicações relataram a presença de faixas com hipossinal em T2, compatíveis com fibras de gaze, sendo estas consideradas imagens clássicas e características de um gossipiboma $^{(\mathbf{1 1 , 1 2})}$. No nosso caso não foi possível identificar cápsula ou faixas de hipossinal em T2 semelhantes a fibras, corroborando a teoria de que a apresentação dos gossipibomas é realmente inconstante. $\mathrm{Na}$ sequência em difusão houve restrição das moléculas de água em alguns focos, traduzindo alto conteúdo proteico no interior, característica já descrita na literatura.

A incidência de corpos estranhos após cirurgias é de $0,01 \%$ a $0,001 \%$. Destes casos, $80 \%$ são gossipibomas, $75 \%$ deles são identificados após cirurgia abdominal ou pélvica $^{(3,7)}$. Porém, esta incidência é subestimada, pois há casos que não são relatados devido a implicações médico-legais.

\section{CONCLUSÃO}

Todas as classes de agentes hemostáticos podem desencadear processos inflamatórios (gossipibomas), porém, estes processos podem mimetizar gliomas de alto grau, radionecrose, abscessos, ou outro tipo de tumor recorrente. Portanto, temos que ficar atentos a esta possibilidade diagnóstica ${ }^{(\mathbf{1})}$.

A identificação e a correta avaliação por imagem desta entidade são de extrema importância, pois definem a conduta cirúrgica quando diagnosticada corretamente.

\section{REFERÊNCIAS}

1. Ribalta T, McCutcheon IE, Neto AG, et al. Textiloma (gossypiboma) mimicking recurrent intracranial tumor. Arch Pathol Lab Med. 2004; 128:749-58.

2. Rajput A, Loud PA, Gibbs JF, et al. Diagnostic challenges in patients with tumors: case 1 . Gossypiboma (foreign body) manifesting 30 years after laparotomy. J Clin Oncol. 2003;21: 3700-1.

3. Kopka L, Fischer U, Gross AJ, et al. CT of retained surgical sponges (textilomas): pitfalls in detection and evaluation. J Comput Assist Tomogr. 1996;20:919-23.

4. Van Goethem JW, Parizel PM, Perdieus D, et al. MR and CT imaging of paraspinal textiloma (gossypiboma). J Comput Assist Tomogr. 1991; 15:1000-3.

5. De Marco JK, McDermott MW, Dillon WP, et al MR appearance of postoperative foreign body granuloma: case report with pathologic confirmation. AJNR Am J Neuroradiol. 1991;12:190-2.

6. Olnick HM, Weens HS, Rogers JV Jr. Radiological diagnosis of retained surgical sponges. JAMA 1955;159:1525-7.

7. Yamato M, Ido K, Izutsu M, et al. CT and ultrasound findings of surgically retained sponges and towels. J Comput Assist Tomogr. 1987;11:10036.

8. Williams RG, Bragg DG, Nelson JA. Gossypiboma: the problem of the retained surgical sponge. Radiology. 1978;129:323-6.

9. Kim HS, Chung TS, Suh SH, et al. MR imaging findings of paravertebral gossypiboma. AJNR Am J Neuroradiol. 2007;28:709-13.

10. Kominami M, Fujikawa A, Tamura T, et al. Retained surgical sponge in the thigh: report of the third known case in the limb. Radiat Med. 2003; 21:220-2.

11. Lo CP, Hsu CC, Chang TH. Gossypiboma of the leg: MR imaging characteristics - a case report. Korean J Radiol. 2003;4:191-3.

12. Nassar I, Mahi M, Semlali S, et al. Textilome intracranien. A propos dun cas. J Neuroradiol. 2004;31:148-9. 